\title{
Near and far transfer: Is music special?
}

\author{
Bigand, E. ${ }^{1,2} \&$ Tillmann B..$^{3,4}$ \\ ${ }^{1}$ University of Burgundy, LEAD, CNRS-UMR 5020, Dijon, France \\ ${ }^{2}$ Institut Universitaire de France \\ ${ }^{3}$ Lyon Neuroscience Research Center; CNRS, UMR5292; INSERM, U1028; Auditory \\ Cognition and Psychoacoustics Team, Lyon, F-69000, France \\ ${ }^{4}$ University Lyon 1, Lyon, F-69000, France
}

Corresponding author:

Emmanuel.Bigand@u-bourgogne.fr

Université de Bourgogne LEAD CNRS UMR 5022

Pôle AAFE - Esplanade Erasme BP 26513

F-21065 DIJON Cedex

Acknowledgements: We thank G. Sala and F. Gobet for our e-mail exchanges related to their meta-analyses and the information provided about their work. The team Auditory Cognition and Psychoacoustics of the Lyon Neuroscience Research Center is part of the framework LabEx CeLyA (“Centre Lyonnais d'Acoustique”, ANR-10-LABX-0060).

\section{Declarations}

- Funding: Auditory Cognition and Psychoacoustics Team is part of the framework LabEx CeLyA (“Centre Lyonnais d'Acoustique”, ANR-10-LABX-0060).

- Conflicts of interest/Competing interests N/A

- Ethics approval N/A

- Consent to participate N/A

- Consent for publication (include appropriate statements): All authors agree for publication.

- Availability of data and materials (data transparency): We used the data file of Sala \& Gobet (2020; https://osf.io/rquye). Our Supplementary Table 1 reproduces the data file of Sala \& Gobet (2020) with additional information about the excluded studies in each of the analyses reported in Tables 1 to 3 . It is openly available in OSF at https://osf.io/w5kx9/.

- Code availability (software application or custom code): We used the program (R script) of Sala \& Gobet (2020; https://osf.io/rquye).

- Authors' contributions (optional: please review the submission guidelines from the journal whether statements are mandatory): Both authors contributed equally to this manuscript. 


\begin{abstract}
Far transfer is rare to occur, and a recent meta-analysis suggests that music is no exception. The overall effect of musical training on cognition was claimed to be null when considering studies with active control groups or implemented randomization procedures (Sala \& Gobet, 2020). Using the authors' data file and program (https://osf.io/rquye), we did not confirm the effect of randomization, and we demonstrated that their conclusion is based in part on the failure to differentiate near and far transfer, with near transfer effect sizes being selectively excluded for the musical training group studies, but not for the active control group studies. Reanalyzing their data file resulted in a significant effect size $(g=.234)$, and also provided new evidence that far-transfer effects of musical training can challenge near-transfer effects of linguistic training. Music is a recreational activity that may be special in allowing for small, but statistically significant far-transfer effects.
\end{abstract}


Recent research investigating neuroplasticity has reignited the debate about near and far transfer, which has a long history in cognitive psychology. The human brain remains plastic throughout life, and this plasticity has important theoretical and social implications for health, wellness and education. The possibility to compensate for aging and to rehabilitate braindamaged patients or children with neurodevelopmental disorders by cognitive stimulation opens new perspectives and leads to understandable enthusiasm. However, this enthusiasm must be qualified: while there is no doubt that specific training improves performance directly linked to the trained skill (referred to as near transfer), it remains unclear whether training in a given task can improve other skills that are not directly related to the training activities (referred to as far transfer). Most cognitive stimulation programs claim to provide far transfer effects, but there are almost as many studies confirming this claim than those denying it. The heterogeneity of the training methods and tests used, the differences in participants, the relatively small sample sizes or the types of implemented control groups undoubtedly contribute to this puzzling panorama. Three questions remain critical: Does far transfer following musical training exist? If yes, is its size of practical interest, and is it actually caused by the training program?

\section{Meta-analyses of cognitive training programs}

Numerous metanalyses have led to strongly moderate the initial enthusiasm about far transfer. Neither working memory training, nor brain training, nor computer games, nor other more playful training, such as chess games, video games or exergames were found to provide far transfer (see Sala \& Gobet, 2019a for a review). A second order meta-analysis of these metaanalyses reported no impact of training on far-transfer measures, regardless of the type of populations and cognitive training programs (Sala et al., 2019). According to the authors, these analyses provide converging evidence that when the allocation procedure (randomization) and the implementation of control groups (i.e., active groups) were controlled for, the far-transfer effects were almost nil, suggesting that previously reported far-transfer effects came mostly from scientifically poor empirical studies. The failure to observe far transfer suggests that "the lack of generalization of skills acquired by training is thus an invariant of human cognition" (Sala et al., 2019; abstract). As a consequence, "researchers and policymakers should seriously consider stopping spending resources for this type of research. Rather than searching for a way to improve overall domain-general cognitive ability, the field should focus on clarifying the domain-specific cognitive correlates underpinning expert performance" (Sala \& Gobet, 2017a, p. 519).

Meta-analyses are a powerful statistical tool, but have some limitations (Borenstein et al., 2009). Although numerous statistical procedures are available, their outcome can be based on several decisions performed without double blindness in an a posteriori process. Our present paper illustrates this limitation by showing how the authors' decisions led to underestimate fartransfer effects for musical training (Sala \& Gobet, 2020). Using open science resources of their publication (https://osf.io/rquye), we revisited their analysis by using their program and data pool and reached a rather different conclusion.

\section{Music and far transfer}

Music is an interesting domain to investigate far transfer. It is a joyful activity, which is easily accessible over lifespan, from young children to the elderly, and for patients with brain deficiency. It can be practiced alone or in a group. Music is also a demanding activity that requires numerous cognitive resources (Patel, 2011). It stimulates brain regions beyond the auditory cortex, including the frontal cortex and the motor cortex, emotional and reward systems as well as socio-affective brain networks. Because the engaged neural network is rather large, music is a good candidate for far-transfer training, which could have "transformational 
power" over the brain (Patel, 2018). Correlational studies have reported brain differences associated to musical training (Herholz \& Zatorre, 2011), and a recent meta-analysis confirmed that musically trained individuals show better memory performance than untrained individuals (Talamini et al., 2017). Correlational studies provide a necessary, but insufficient demonstration for a causal relationship. The repetition of a demanding task over months and even years could plausibly shape the brain, but an alternative explanation is that only the smarter individuals manage to pursue this training. Put differently, music does not make people smarter, but smarter individuals are more likely to start to learn and to succeed to play music (Schellenberg, 2020). Experimental studies implementing musical training in a longitudinal approach have led to disparate findings (Sala \& Gobet, 2017b). Once again, meta-analyses seem promising to further assess whether musical training might be inducing far transfer effects.

To the best of our knowledge, seven published meta-analyses have addressed this issue up to now. The first three have included rather small sets of studies and effect sizes (Vaughn, 2000; Hetland \& Winner, 2001; Gordon et al., 2015). A more elaborated meta-analysis was performed by Sala and Gobet (2017b) with 38 studies investigating 3- to 16-year old children, leading to the inclusion of 118 effect sizes and 3085 participants. This same pool of studies (minus two studies) was reanalyzed in a second-order meta-analysis (Sala et al., 2019a). The majority of these studies was then combined with more recent studies in Sala and Gobet (2019b), leading to 43 studies, 204 effects size and 3,780 participants. Finally, a selection of these studies was combined with eleven new studies in a multilevel meta-analysis, including 54 studies, 254 effect sizes and 6,984 participants, which will be the focus of our present paper (Sala \& Gobet, 2020; referred to as S\&G 2020 hereafter). Another recent meta-analysis (Cooper, 2020) was run with 21 studies and 100 effect sizes (numerous had also been included in Sala \& Gobet, 2017b, 2020). In contrast to Sala and Gobet (2017b, 2019b, 2020), Cooper's (2020) meta-analysis reported a moderate overall effect of musical training for both active and non-active control group studies $(g=.28)$, but this effect failed to be significant for studies done in a laboratory setting instead of classroom or community center settings.

\section{Sala \& Gobet's (2020) meta-analyses of music training programs}

The present paper focuses on S\&G2020's multilevel meta-analysis approach. Several moderators relative to randomization, type of control, baseline differences, age, duration of training and type of outcome measures were included. These outcome measures were organized into four categories: non-verbal ability (fluid reasoning, mathematical and spatial skills), verbal ability (vocabulary and reading skills, phonological processing), memory (short-term/workingmemory tasks) and speed (processing speed and inhibition tasks). Three modeling approaches were used, notably the robust variance estimation (RVE), a random effect model (RE) and Bayesian analysis. Only far transfer tests following musical training run with typically developing, 3- to 16-year-old children were considered.

As a main outcome, S\&G2020 reported an overall impact of music training programs on cognitive and academic outcomes $(g=0.184, p<.001)$ that dropped close to zero when either only the active control group studies $(g=0.056 ; p=.350)$ or the randomized non-active control group studies ( $g=0.064, p=.381$ ) were considered separately (see Table 1 for details). S\&G2020 concluded that when confounding factors, such as type of controls or the lack of random assignment of participants to the groups, were neutralized, the overall effect of music training was null. Neither age, duration of training, and outcome measures were found to have a significant contribution. Accordingly, "researchers' optimism about the benefits of music training is empirically unjustified and stems from misinterpretation of the empirical data and, possibly, confirmation bias" ( $S \& G, 2020$, page 1429). This finding was considered to be consistent with their previous conclusions, summarized as "Music is over" (Sala \& Gobet, 2017b) and "Elvis has left the building" (Sala \& Gobet, 2019b). For the authors, "the obvious 
practical implication is that music training should not be used as a tool for cognitive enhancement" (Sala \& Gobet, 2019b, page 991) and "Educators and policymakers should be aware that music training provides no benefits on non-music-related cognitive or academic skills" (ibid.).

We here propose to reconsider their conducted meta-analysis and its swift conclusions in three steps. We focus first on the potential influence of randomization. S\&G2020 found a significant effect of this factor, but Sala and Gobet (2019b) and Sala and Gobet (2017b, after their sensitivity analysis) did not. We then demonstrate for S\&G2020 that the active control group studies instilled an unfair comparison, notably by including near transfer effects in the control group studies, but only far transfer effects in the musical training studies. Finally, using S\&G2020's data file and R program (https://osf.io/rquye), we ran a set of meta-analyses that removed both concerns aiming to have a more appropriate estimation of the effect of music training (here based on the studies included in their data file).

\section{Revisiting Sala and Gobet's (2020) meta-analyses on musical training}

\subsection{Randomization}

One of the two main conclusions of S\&G2020 was that the observed effect of musical training vanishes when only randomized studies were considered. Randomization was not a significant moderator in their previous meta-analysis (Sala \& Gobet, 2019b), which involved 204 of the 254 effect sizes of S\&G2020. Randomization was also not a significant moderator in the main analysis of $\mathrm{S} \& \mathrm{G} 2020$ ( $p=.518^{1}$, based on all effect sizes). In contrast to Sala and Gobet (2019b), S\&G2020 ran a two-step sensitivity analysis. After the first step of the sensitivity analysis of $\mathrm{S} \& \mathrm{G} 2020$, randomization was not a significant moderator $\left(p=.693^{1}\right)$, but type of control was (as in the main analysis). This led S\&G2020 to perform the subgroup analyses: For the non-active control group studies, the effect size remained significant ( $g=$ $0.226 ; p<.001$, see Table 1 middle), and no moderator analysis was reported at that point. When running a moderator analysis with their program, it revealed that randomization was not a significant moderator $(p=.480)$. At that stage, the second step of S\&G2020's sensitivity analysis intervened: S\&G2020 ran an influential case analysis and wrote "Five effect sizes were found to be significantly inflating the true heterogeneity" (p. 1435). Removing these values resulted in the reduced $g$ of 0.181 (Table 1, right), and a moderator analysis was run. Although not explicitly written in manuscript, this moderator analysis was slightly different from that performed for the other analyses. Instead of running one moderator analysis with all moderators (here randomization, baseline, age), the program (see lines 737-739) reveals that S\&G2020 ran here three separate moderator analyses, each one with one moderator. With this change in model, randomization was a significant moderator, $p=.042$. However, when we ran one moderator analysis with the three moderators (similar to the other moderator analyses performed by S\&G2020, see lines 579 and 665 of their program), the contribution of randomization did not reach significance $(p=.08)$. When performing the analysis with their program, we also observed that the influential case analysis suggests nine influential effect sizes for this data set, and not five as stated by S\&G2020 (p. 1435). When all nine influential cases were removed, the effect size for the non-active control group studies remained significant ( $g$ $=0.203 ; p<.0001$; see Table 2 left) and, most importantly, heterogeneity dropped to 0 (RVE: $I^{2}$ and $\tau^{2}$ ), indicating that these additional four influential cases were actually increasing heterogeneity. Once again randomization was not a significant moderator ( $p=.194$ or $p=.158$ when running one or three moderator analyses, respectively).

In sum, these findings suggest that randomization is not a robust moderator, and was

\footnotetext{
${ }^{1}$ The authors ran this moderator analysis, but the manuscript did not list this p-value, we recalculated it with the open source program provided by S\&G2020.
} 
obtained by S\&G2020 via a two-step sensitivity analysis, with the second step applying the influential case analysis only to the non-active control group studies, by removing only five influential cases (out of nine) and changing the implementation of the moderator analysis. In contrast to S\&G2020, Sala and Gobet (2019b) ran a simpler, one-step process: an influential case analysis was run on all studies of the main analysis and did not find evidence for a significant influence of the moderator randomization. We applied this one-step process to the present data file of S\&G2020 (i.e., the full data set as used in S\&G2020's main analysis). The influential case study revealed 16 influential effect sizes (see Supplementary Table 1 for details of studies excluded for all analyses reported in this manuscript, available at https://osf.io/w5kx9/). Without them, the effect size was significant $(p<.0001$; see Table 2 right), and the moderators randomization and type of control did not reach significance ( $p=$ .476 and $p=.064$ ). For comparison purpose with $\mathrm{S} \& \mathrm{G} 2020$, we nevertheless ran the separate analysis for non-active control group studies, and the effect size remained significant ( $g=0.202$; $p<.0001$; see Table 2, right), the heterogeneity was $0\left(\mathrm{I}^{2}\right.$ and $\left.\tau^{2}\right)$ and randomization was not a significant moderator ( $p=.185$ or $p=.157$ when running one or three moderator analyses, respectively). (See Supplementary Material for Bayesian analyses). In agreement with the previous findings of Sala and Gobet (2019b), our reanalysis here provides converging evidence that randomization is not a significant moderator.

\subsection{Near vs. far transfer in control vs. experimental training programs}

The second main conclusion of S\&G2020 is that the effect of musical training is null for active control group studies. In the following, we demonstrate that this conclusion is based on their failure to differentiate far transfer from near transfer. When active control groups perform sport, computer or video game activities, the various pre- and post-test tasks measure far-transfer effects, as for the experimental musical training groups. However, when the control group follows drama lessons and is evaluated, just like the musical training group, on linguistic performance, then this raises the question of the relevance of the active control group and its equidistance to the tests. Given that drama stimulates different facets of linguistic abilities, drama training is closer to the linguistic target tasks than is musical training, and we know from a previous meta-analysis that a group with classroom drama training (i.e., enacting text) outperforms an active control group (passive reading) on different verbal skills, such as writing, story understanding and recall as well as oral understanding (Hetland \& Winner 2001). The concern raised here becomes even more important when the active control group is directly trained on linguistic tasks that are similar to the target tasks used in pre- and post-tests. For instance, when the control group receives phonological training and is tested on phonological awareness, or when the control group is trained on reading and evaluated for reading, the active control group is tested for near transfer, while the musical training group is tested for far transfer (with these same language tests). This results in a somewhat biased or unfair comparison, notably with effect sizes having a different meaning here than do effect sizes in an equidistant control training implementation. In such an unequal comparison, an effect size close to zero does not mean that musical training does not create transfer effects. It indicates that musical training creates far-transfer effects that are not stronger than the near-transfer effects instilled by the given control trainings. S\&G2020 were well aware of differences between far transfer and near transfer, and they correctly removed all effect sizes associated with musical tests (e.g., pitch, rhythm) and even environmental sound discrimination tests. However, they did not apply the same caution to the active control group studies. This unequal treatment leads to underestimate the effect of musical training in the analyses ${ }^{2}$.

\footnotetext{
${ }^{2}$ One might argue that phonological processing may benefit from musical training because musical activities train listening skills. However, phonological tests tap into processes that go beyond sound discrimination and that have no equivalent in the musical training program, such as for example rhyme detection, phoneme-grapheme correspondence or phoneme deletion, which requires participants to remove one phoneme from a nonword to create a word. As a consequence, phonological training is necessarily closer to the phonological tasks than is
} 
Aiming to assess the extent of this underestimation, we removed from S\&G2020's data file the 21 effect sizes that are related to the most unbalanced comparisons. As the boundary between "near" and "far" transfer might be a matter of debate, we excluded only effect sizes that tap into highly similar constructs in training and test in the control group: 18 effect sizes were related to the comparison with an active control group receiving phonological training and being tested on phonological processing, two effect sizes were related to the comparison with an active control group receiving a reading intervention and being tested on reading, and one effect size was related to the comparison with an active control group receiving visual art lessons and being tested on visual form analysis (see Supplementary Table 1 for details). All other effect sizes, including those linked to drama or dance remained included. Using the program of S\&G2020, we observed comparable (or even slightly increased) effect sizes for this reduced data set (RVE: $g=0.208 ; p<.0001$; RE: $g=0.195 ; p<.0001$; see Table 3 left, for details), with similar heterogeneity as in the main analysis of the authors (see Table 1 left). As the authors did in the main analysis, we ran a moderator analysis with type of control, randomization, baseline and age as moderators, but neither randomization nor type of control was significant $(p=.610$ and $p=.193$, respectively). (See Supplementary Material for complementary analyses)

To further investigate whether near-transfer effects induced by linguistic training (and art lessons for one measure, i.e., the set of active control training studies described above) was significantly stronger than far-transfer effects induced by musical training, a subgroup analysis was performed on the 21 removed effect sizes (corresponding to eight studies). We observed an effect size of $g=-0.126\left(S E=.071 ; 95 \%\right.$ CI $[-0.350 ; 0.099] ; \mathrm{df}=3.01 ; p=.17 ; \mathrm{I}^{2}=0 ; \tau^{2}=$ 0 ) with the RVE model, and $g=-0.117\left(S E=.107 ; p=.275 ; \tau^{2}=0\right)$ for the RE model. This absence of difference was further supported by Bayesian analyses; the Bayes factor $\left(B F_{g}=\right.$ 0.447) provided some evidence that $g$ was more likely be null than non-null (i.e., H0 almost 2.24 times more likely to be true than H1) (see Supplementary Material for details) ${ }^{3}$. This finding provides new evidence suggesting that far-transfer effects induced by musical training could even compete with near-transfer effects induced by linguistic training (and art lessons for one measure).

\subsection{Meta-analysis without post-test-only studies}

A further concern with S\&G2020's sensitivity analysis was to include studies that did not report pretest measures of the targeted tests. In these cases, the program of S\&G2020 assumes that experimental and control groups did not differ at pre-test (coded as a difference of 0 in the baseline moderator), which is certainly unlikely, in particular in developmental psychology. In another meta-analysis run with a similar set of studies, the authors had excluded post-test-only studies (Sala et al., 2019), and this was done also by Gordon et al. (2015). We thus applied this rationale to the present data set (i.e., removing all effect sizes without pre-test measures from the data file of S\&G2020), while still focusing on studies testing for far-transfer effects. This analysis confirmed a significant overall effect size (RVE: $g=0.243 ; p<.0001 \mathrm{RE}$ : $g=.226 ; p<.0001$; see Table 3, middle), and neither randomization nor type of control was a significant moderator ( $p=.676$ and $p=.181$, respectively). (See Supplementary Material for

\footnotetext{
musical training.
}

\footnotetext{
${ }^{3}$ One of the included studies (Herrera et al., 2011) used a phonological outcome measure and compared experimental and control groups that both received phonological training (i.e., near transfer), with the experimental group performing this training in music. In response to the argumentation that this study should not have been included here, we reran the analysis by excluding the eight effect sizes of this study. The results of this additional analysis confirmed the previous finding, notably an effect size of $\mathrm{g}=-0.126(\mathrm{SE}=.076 ; 95 \% \mathrm{CI}[-0.385 ; 0.131]$; $\mathrm{df}=$ $\left.2.68 ; \mathrm{p}=.20 ; \mathrm{I}_{2}=0 ; \tau_{2}=0\right)$ with the RVE model, and $\mathrm{g}=-0.118\left(\mathrm{SE}=.110 ; \mathrm{p}=.287 ; \tau_{2}=0\right)$ for the RE model. This absence of difference was further supported by Bayesian analyses; the Bayes factor $\left(\mathrm{BF}_{\mathrm{g}}=0.383\right)$ provided evidence that $\mathrm{g}$ was more likely be null than non-null (see Supplementary Material for details)
} 
complementary analyses).

We then applied an influential case analysis on the effect sizes of this data set, following the procedure of Sala and Gobet (2019b). This influential case analysis led us to remove seven effect sizes (i.e., six were positive), which reduced heterogeneity (see Table 3 right for details) ${ }^{4}$. The overall effect size was significant (RVE: $g=0.234 ; p<.0001$; RE: $g=0.213 ; p<.0001$ ), and again, the moderators type of control and randomization were not significant $(p=.163$ and $p=.319$, respectively). (See Supplementary Material for complementary analyses).

Our finding is consistent with two recent meta-analyses reporting a significant effect size of musical training, albeit slightly stronger, $g=.26$ in Román-Caballero et al. (2021), and $g=.28$ in Cooper (2020). These small differences in effect sizes might be explained by several minor decisions of the different authors about potential selection or merging of effect sizes for a given study. For example, Cooper (2020) included six positive effect sizes for Bilhartz et al. (1999; notably $d=.37, d=.56, d=.68, d=.70, d=.75, d=.78)$, while S\&G2020 included only one effect size $(d=.19)$. Similarly, for the study of Costa Giomi (2004), three positive effect sizes were included by Roman-Caballero et al. (2021, notably $d=.34, d=.40$. and $d=$ $.53)$, while S\&G2020 included only one $(d=.209)$. On the contrary, S\&G2020 included all 26 effect sizes of Rickard et al. (2012), who failed to find an effect of music training, while Cooper (2020) included only ten. These observations suggest that S\&G2020 favored an approach with more conservatism, which is further supported by other changes between Sala and Gobet (2017b) and S\&G2020 (i.e., from a set of 13 effect sizes, ten effect sizes decreased and two positive effect sizes were excluded in S\&G2020). All of these points might contribute to underestimate the potential effect size of music training in S\&G2020, and when revisiting their data file, we inherited this tendency.

This said, it might be argued that effect sizes ranging from .234 to .28 remain small according to Hattie's (2008) barometer of influence. In his book, Hattie (2008) analyzed more than 800 meta-analyses and reported that the median value of intervention effect sizes in education is 0.40 . One simplistic way to understand this contribution would be to recommend that all effects below this value should be ignored, as $50 \%$ of all interventions obtained at least such an effect. Along this line, the effects of music training on cognitive abilities could be considered as too small to be of any practical use, a view consistent with S\&G2020's conclusion. However, according to Hattie (2008), this effect size of .40 "is not a magic number that should become like a $p<.05$ cut-off point" (page 17). "Effect lower than $d=.40$ can be regarded as a need of more consideration, although it is not as simple as saying that all effect below $d=.40$ are not worth having" (page 16). "There are many examples that show small effects may be important" (page 9) and Hattie insists on the fact that the value of an effect size also depends on the cost of its implementation. For instance, the effect of homework, which is typically $d=.29$ according to Hattie (page 234), is of interest because of its low cost of implementation. A similar situation occurs with music training, which is a recreational activity with low cost and with effect sizes likely to be in the same range as visual/audio visual learning $(d=.22$, page 229$)$ or programed instruction $(d=.24$, page 231$)$ and larger than effect sizes of extra curricula activities $(d=.17$, page 159$)$, sport $(d=.10)$ and numerous teaching approaches explicitly designed to improve achievement, such as "mentoring" $(d=.15$, page 188), aptitude treatment interaction $(d=.19$, page 194$)$, problembased learning $(d=.15$ page 211$)$, web-based learning $(d=.18$, page 227$)$, or home school programs $(d=.16$, page 234). A more recent publication, which involved 1200 metaanalyses (Hattie, 2015) even reported an effect size of .37 for music-based programs, which thus placed music at rank 94 among the 195 variables that influence school achievement.

\footnotetext{
${ }^{4}$ Note that in comparison to the data set of S\&G2020 used in their main analyses, the data set used here was overall reduced by as many effect sizes in randomized (26) than in non-randomized (27) studies.
} 


\section{Conclusion}

Over the last five years, Sala and Gobet have published several meta-analyses providing converging evidence that cognitive training does not enhance general cognition (see Sala \& Gobet 2019a for a review). Their finding about music training fits well with this claim (Sala \& Gobet, 2017b; 2019b; 2020) and has led them to conclude that "researchers' optimism about the benefits of music training is empirically unjustified and stems from misinterpretation of the empirical data and, possibly, confirmation bias" (Sala \& Gobet, 2020, page 1429).

Thanks to the resources made available by S\&G2020 within the Open Science Framework related to their manuscript, we revisited their meta-analysis of 2020 . We provided some evidence that their findings are based on decisions that led them to underestimate potential far-transfer effects created by music training. Our findings show the importance to test experimental and control groups for far transfer. Without such a fair comparison, the effect of music training is underestimated. As all meta-analyses of Sala and Gobet on musical training included studies with an unbalanced far- versus near-transfer comparison, which is unfavorable to musical training, their conclusions need to be reevaluated. This issue also applies to Sala et al.'s (2019) second-order meta-analysis that combined their meta-analysis on music training (Sala \& Gobet, 2017b) with meta-analyses on other cognitive training types. Revisiting this second order meta-analysis is now needed to further evaluate whether music might actually be a special case allowing for far transfer.

Our findings converge with those of other recent meta-analyses towards a consistent conclusion for the first two questions we raised in our introductory section: yes, there is a significant effect of musical training on general cognition, and it can be considered of practical interest, even if its size remains small according to Hattie (2008)'s barometer of influence. It would not seem reasonable to expect that a couple of hours of musical training per week could place music among the most efficient educational interventions (i.e., those with effect sizes $d$ superior to .40). The fact that this recreational activity of low implementation cost succeeds to create significant far-transfer effects to general cognition is of interest for cognitive psychology, and it may have practical implications for educational science. The third question raises the need to specify whether music may cause these benefits. Revisiting S\&G2020 data file here supported a general effect of music training, which is not significantly modulated by randomization or type of control. A causal interpretation thus cannot be rejected, even though this point needs further new studies that should rigorously control for randomization, fair active control group comparisons, the inclusion of both pre- and post-test measurements as well as the measurement of IQ at baseline. For now, all findings together lead us to conclude that music is not over and Elvis is still on stage. 


\section{Data availability}

The data set and R program used in the present manuscript are those of Sala \& Gobet (2020) who made them openly available in OSF at https://osf.io/rquye/. The Supplementary Table 1 reproduces the data file of Sala \& Gobet (2020) with additional information about the excluded studies in each of the analyses reported in Tables 1 to 3. It is openly available in OSF at https://osf.io/w5kx9/. 


\section{References}

Aleman, X., Duryea, S., Guerra, N. G., McEwan, P. J., Muñoz, R., Stampini, M., \& Williamson, A. A. (2017). The effects of musical training on child development: A randomized trial of El Sistema in Venezuela. Prevention Science, 18, 865-878. DOI: 10. 1007/s11121-0160727-3

Bilhartz, T. D., Bruhn, R. A., \& Olson, J. E. (1999). The effect of early music training on child cognitive development. Journal of Applied Developmental Psychology, 20, 615-636.

Borenstein, M., Hedges, L. V., Higgins, J. P. T. \& Rothstein, H. R. (2009) Introduction to Meta-Analysis. John Wiley \& Sons.

Cooper, P. K. (2020). It's all in your head: A meta-analysis on the effects of music training on cognitive measures in school children. International Journal of Music Education. 38:3, 321336 DOI: $10.1177 / 0255761419881495$.

Costa-Giomi, E. (2004). Effects of three years of piano instruction on children's academic achievement, school performance and self-esteem. Psychology of Music, 32(2), 139-152. https://doi.org/10.1177/0305735604041491

Gordon, R. L., Fehd, H. M., \& McCandliss, B. D. (2015). Does music training enhance literacy skills? A meta-analysis. Frontiers in Psychology, 6:1777. DOI: 10.3389/fpsyg.2015.01777

Hattie, J. A. C. (2008). Visible Learning: A Synthesis of Over 800 Meta-Analyses Relating to Achievement. Routledge, Taylor and Francis Group. London.

Hattie, J. (2015). The Applicability of Visible Learning to Higher Education, Scholarship of Teaching and Learning in Psychology, 1 (1), 79-91. https://doi.org/10.1037/st10000021

Haywood, S., Griggs, J., Lloyd, C., Morris, S., Kiss, Z., \& Skipp, A. (2015). Creative futures: Act, sing, play. Evaluation report and executive summary. London: Educational Endowment Foundation.

Herholz, S. \& Zatorre, R. (2011) Musical training as a framework for brain plasticity: behavior, function, and strucutre. Neuron, 76: 3, 486-502 DOI: 10.1016/j.neuron.2012.10.011

Hetland, L., \& Winner, E. (2001). The arts and academic achievement: What the evidence shows. Arts Education Policy Review, 102, 3e6. DOI: 10. 1080/10632910109600008.

Maassen, E., van Assen, M. A. L. M., Nuijten, M.B., Olsson-Collentine, A. \& Wicherts, J.M. (2020.) Reproducibility of individual effect sizes in meta-analyses in psychology. PLoS ONE 15(5): e0233107. https://doi.org/10.1371/journal.pone.0233107

Patel, A. D. (2011). Why would musical training benefit the neural encoding of speech? The OPERA hypothesis. Frontiers in Psychology, 2, 142. DOI: 10.3389/fpsyg.2011.00142

Patel, A.D. (2018) Music as a Transformative Technology of the Mind: An Update (pp. 113126), In H. Honing (Ed.), The origin of musicality, MIT Press: Cambridge MA.

Patscheke, H., Degé, F., \& Schwarzer, G. (2019). The effects of training in rhythm and pitch on phonological awareness in four- to six-year-old children. Psychology of Music, 47, 376-391. doi:10.1177/0305735618756763

Rickard, N. S., Bambrick, C. J., \& Gill, A. (2012). Absence of widespread psychosocial and cognitive effects of school-based music instruction in 10-13-year-old students. International Journal of Music Education, 30, 57-78. DOI: 10.1177/0255761411431399

Roden, I., Kreutz, G., \& Bongard, S. (2012). Effects of a school-based instrumental music program on verbal and visual memory in primary school children: A longitudinal study. Frontiers in Psychology, 3:572. doi:10.3389/fpsyg.2012.00572

Roden, I., Konen, T., Bongard, S., Frankenberg, E., Friedrich, E. K., \& Kreutz, G. (2014). Effects of music training on attention, processing speed and cognitive music abilities - Findings from a longitudinal study. Applied Cognitive Psychology, 28, 545-557. DOI: 10.1002/acp.3034

Román-Caballero, R., Vadillo, M. A., Trainor, L., \& Lupiáñez, J. (2021, April 13). Please 
Don't Stop the Music: A Meta-Analysis of the Benefits of Learning to Play an Instrument on Cognitive and Academic Skills. https://doi.org/10.31234/osf.io/4bm8v

Sala, G., \& Gobet, F., (2017a). Does Far Transfer Exist? Negative Evidence From Chess, Music, and Working Memory Training, Current Directions in Psychological Science; 26(6) 515-520 DOI: 10.1177/0963721417712760

Sala, G., \& Gobet, F. (2017b). When the music's over. Does music skill transfer to children's and young adolescents' cognitive and academ- ic skills? A meta-analysis. Educational Research Review, 20, 55-67. DOI: 10.1016/j.edurev.2016.11.005

Sala, G., \& Gobet, F. (2019a). Cognitive training does not enhance general cognition. Trends in Cognitive Sciences, 23, 9-20. DOI: 10. 1016/j.tics.2018.10.004

Sala, G., \& Gobet, F. (2019b). Elvis has left the building: Correlational but not causal relationship between music skill and cognitive ability. Proceedings of the $41^{\text {st }}$ Annual Meeting of the Cognitive Science Society (pp. 987-993), Montreal, Canada.

Sala, G., N. D. Aksayli, K. S. Tatlidil, T. Tatsumi, Y. Gondo \&F. Gobet (2019) Near and Far Transfer in Cognitive Training: A Second-Order Meta-Analysis. Collabra: Psychology, 5(1): 18. DOI: 10.1525/collabra.203

Sala, G. \& Gobet, F. (2020). Cognitive and academic benefits of music training with children: A multilevel meta-analysis , Memory \& Cognition, 48:1429-1441; DOI: 10.3758/s13421-020-01060-2

Schellenberg, E. G. (2020). Music Training, Individual Differences, and Plasticity (pp. 415442), In M.S.C. Thomas, D. Mareschal \& I. Dumontheil, Educational Neuroscience, Frontiers of Developmental Science, Routledge Taylor \& Francis Group: New York and London.

Talamini, F., Altoè, G., Carretti, B., \& Grassi, M. (2017). Musicians have better memory than nonmusicians: A meta-analysis. PLoS One 12: e0186773. DOI: 10.1371/journal.pone.0186773

Vaughn, K. (2000). Music and mathematics: Modest support for the oft-claimed relationship. Journal of Aesthetic Education, 34, 149e166. DOI: 10.2307/3333641. 
Table 1. Summary presentation of the analyses presented in Sala \& Gobet $(2020$, p. 1435f)

\begin{tabular}{|c|c|c|c|}
\hline & Main analyses & $\begin{array}{l}\text { Senstivity analysis Step } 1 \\
\quad(3 \text { studies removed })\end{array}$ & \\
\hline & $m=54 ; k=254$ & $m=51 ; k=235$ & \\
\hline RVE & $\begin{aligned} \mathbf{g}=\mathbf{0 . 1 8 4} ; \mathrm{SE}=0.041 ; \mathbf{p}<\mathbf{. 0 0 1} \\
\mathrm{df}=38.36 ; 95 \% \text { CI }[0.101 ; 0.268] \\
\boldsymbol{\tau}^{\mathbf{2}}=\mathbf{0 . 0 4 1} ; \mathbf{I}^{\mathbf{2}}=\mathbf{4 3 . 1 6 \%}\end{aligned}$ & $\begin{array}{c}\mathbf{g}=\mathbf{0 . 1 6 6} ; \text { SE }=0.041 ; \mathbf{p}<\mathbf{. 0 0 1} \\
\mathrm{df}=34.9 ; 95 \% \text { CI }[0.083 ; 0.249] \\
\boldsymbol{\tau}^{2}=\mathbf{0 . 0 3 6} ; \mathbf{I}^{\mathbf{2}}=\mathbf{4 0 . 6 2 \%}\end{array}$ & \\
\hline \multirow[t]{3}{*}{$\mathrm{RE}$} & $\begin{array}{c}\mathrm{g}=\mathbf{0 . 1 7 6} ; \mathrm{SE}=0.037 ; \mathbf{p}<.001 \\
\boldsymbol{\tau}^{2}=\mathbf{0 . 0 3 3}\end{array}$ & $\begin{array}{c}\mathrm{g}=0.149 ; \mathrm{SE}=0.035 ; \mathrm{p}<.001 \\
\boldsymbol{\tau}^{2}=\mathbf{0 . 0 2 4}\end{array}$ & \\
\hline & Non active control group studies & Non active control group studies: & $\begin{array}{l}\text { Step } 2 \text { (5 influential cases removed) } \\
\text { Non active control group studies: }\end{array}$ \\
\hline & $m=41 ; k=144$ & $m=40 ; k=139$ & $m=39 ; k=134$ \\
\hline RVE & $\begin{array}{c}\mathbf{g}=\mathbf{0 . 2 2 8} ; \mathrm{SE}=0.045 ; \mathbf{p}<\mathbf{. 0 0 1} \\
\mathrm{df}=30.1 ; 95 \% \text { CI }[0.137 ; 0.320] \\
\boldsymbol{\tau}^{2}=\mathbf{0 . 0 4 2} ; \mathbf{I}^{\mathbf{2}}=\mathbf{4 3 . 1 1 \%}\end{array}$ & $\begin{array}{c}\mathbf{g}=\mathbf{0 . 2 2 6} ; \mathrm{SE}=0.045 ; \mathbf{p}<. \mathbf{0 0 1} \\
\mathrm{df}=29.2 ; 95 \% \mathrm{CI}[0.133 ; 0.319] \\
\boldsymbol{\tau}^{\mathbf{2}}=\mathbf{0 . 0 4 1} ; \mathbf{I}^{\mathbf{2}}=\mathbf{4 2 . 9 6 \%}\end{array}$ & $\begin{array}{c}\mathbf{g}=\mathbf{0 . 1 8 1} ; \mathrm{SE}=0.042 ; \mathbf{p}<. \mathbf{0 0 1} \\
\mathrm{df}=21.9 ; 95 \% \text { CI }[0.093 ; 0.268] \\
\boldsymbol{\tau}^{\mathbf{2}}=\mathbf{0 . 0 1 8} ; \mathbf{I}^{\mathbf{2}}=\mathbf{2 4 . 9 2 \%}\end{array}$ \\
\hline $\mathrm{RE}$ & $\begin{array}{c}\mathrm{g}=\mathbf{0 . 2 0 1} ; \mathrm{SE}=0.041 ; \mathbf{p}<.001 \\
\boldsymbol{\tau}^{2}=\mathbf{0 . 0 2 3}\end{array}$ & $\begin{array}{cl}\mathbf{g}=\mathbf{0 . 2 0 0} ; & \mathrm{SE}=0.041 ; \mathbf{p}<.001 \\
& \boldsymbol{\tau}^{2}=\mathbf{0 . 0 2 4}\end{array}$ & $\begin{array}{cl}\mathbf{g}=\mathbf{0 . 1 6 1} ; & \mathrm{SE}=0.037 ; \mathbf{p}<.001 \\
& \tau^{2}=\mathbf{0 . 0 1 3}\end{array}$ \\
\hline \multirow[t]{3}{*}{$\begin{array}{c}\text { Moderator } \\
\text { Randomization }\end{array}$} & $p=.487^{1}$ & $p=.480^{l}$ & $\begin{array}{c}p=.042^{2} \\
\text { Non-randomized studies } \\
\mathbf{g}=\mathbf{0 . 2 4 6} ; \mathrm{SE}=0.049 ; \mathbf{p}<\mathbf{. 0 0 1} \\
95 \% \mathrm{CI}[0.140 ; 0.352] \\
\text { Randomized studies } \\
\mathbf{g}=\mathbf{0 . 0 6 4} ; \mathrm{SE}=0.065 ; \mathbf{p}=\mathbf{. 3 8 1} \\
95 \% \text { CI }[-0.116 ; 0.244]\end{array}$ \\
\hline & Active control group studies & Active control group studies & \\
\hline & $m=23 ; k=110$ & $m=20 ; k=96$ & \\
\hline RVE & $\begin{array}{c}\mathbf{g}=\mathbf{0 . 0 5 6} ; \mathrm{SE}=0.058 ; \mathbf{p}=\mathbf{0 . 3 5 0} \\
\mathrm{df}=12.6 ; 95 \% \text { CI }[-0.069 ; 0.182] \\
\boldsymbol{\tau}^{\mathbf{2}}=\mathbf{0 . 0 2 5} ; \mathbf{I}^{\mathbf{2}}=\mathbf{2 3 . 1 0 \%}\end{array}$ & $\begin{array}{c}\mathbf{g}=\mathbf{- 0 . 0 2 1} ; \mathrm{SE}=0.032 ; \mathbf{p}=\mathbf{. 5 5 8} \\
\mathrm{df}=4.2 ; 95 \% \text { CI }[-0.109 ; 0.068] \\
\boldsymbol{\tau}^{2}=\mathbf{0} ; \mathbf{I}^{\mathbf{2}}=\mathbf{0}\end{array}$ & \\
\hline $\mathrm{RE}$ & $\begin{array}{c}\mathrm{g}=\mathbf{0 . 0 9 0} ; \mathrm{SE}=0.060 ; \mathbf{p}=\mathbf{. 1 3 6} \\
\tau^{2}=\mathbf{0 . 0 3 2}\end{array}$ & $\begin{array}{c}\mathbf{g}=\mathbf{- 0 . 0 1 0} ; \mathrm{SE}=0.035 ; \mathbf{p}=\mathbf{. 7 8 7} \\
\tau^{2}=\mathbf{0}\end{array}$ & \\
\hline
\end{tabular}


Note: $m$ refers to the number of studies, $k$ refers to the number of effect sizes. We used S\&G2020's R script and data file, available at https://osf.io/rquye. Supplementary Table 1 lists the studies excluded at the various analysis steps.

${ }^{1}$ We run this moderator analysis (not reported in the manuscript) with the authors' program. It included the three moderators Randomization,

Baseline and Age.

${ }^{2}$ The authors' program showed (lines 737-739) that the authors ran three separate analyses, one for each moderator: 1) randomization, 2) baseline, 3) age. When we run one moderator analysis with the three moderators, similar to the other moderator analyses performed before by the authors (lines 579 and 665), the influence of the moderator Randomization was at $p=.08$. 
Table 2: Alternative sensitivity analyses of Sala \& Gobet's data set, see main text for details (see Section 4.1)

\begin{tabular}{|c|c|c|}
\hline & Sensitivity analysis Step 2 & $\begin{array}{l}\text { Influential case analysis on all } \\
\text { studies (main analysis data) }\end{array}$ \\
\hline & & $m=51 ; k=238$ \\
\hline RVE & & $\begin{aligned} \mathbf{g}=\mathbf{0 . 1 7 1} ; \mathrm{SE}=0.038 ; \mathbf{p}<\mathbf{. 0 0 0 1} \\
\mathrm{df}=33.40 ; 95 \% \text { CI }[0.095 ; 0.248] \\
\boldsymbol{\tau}^{\mathbf{2}}=\mathbf{0 . 0 1 5} ; \mathbf{I}^{\mathbf{2}}=\mathbf{1 4 . 4 1 \%}\end{aligned}$ \\
\hline \multirow[t]{3}{*}{$\mathrm{RE}$} & & $\begin{aligned} \mathbf{g}=\mathbf{0 . 1 5 2} ; \mathrm{SE} & =0.032 ; \mathrm{p}<.0001 \\
\boldsymbol{\tau}^{2} & =\mathbf{. 0 1 8}\end{aligned}$ \\
\hline & $\begin{array}{c}\text { Non active control group studies: } \\
\text { Step } 2 \text { (all } 9 \text { influential cases } \\
\text { removed) }\end{array}$ & $\begin{array}{c}\text { Non active control group } \\
\text { studies }\end{array}$ \\
\hline & $m=38 ; k=130$ & $m=39 ; k=134$ \\
\hline RVE & $\begin{array}{c}\mathbf{g}=\mathbf{0 . 2 0 3} ; \mathrm{SE}=0.036 ; \mathbf{p}<. \mathbf{0 0 0 1} \\
\mathrm{df}=19.77 ; 95 \% \text { CI }[0.128 ; 0.278] \\
\boldsymbol{\tau}^{\mathbf{2}}=\mathbf{0} ; \mathbf{I}^{\mathbf{2}}=\mathbf{0 \%}\end{array}$ & $\begin{array}{c}\mathbf{g}=\mathbf{0 . 2 0 2} ; \mathrm{SE}=0.035 ; \mathbf{p}<\mathbf{. 0 0 0 1} \\
\mathrm{df}=20.18 ; 95 \% \text { CI }[0.129 ; 0.276] \\
\boldsymbol{\tau}^{\mathbf{2}}=\mathbf{0} ; \mathbf{I}^{\mathbf{2}}=\mathbf{0} \%\end{array}$ \\
\hline $\mathrm{RE}$ & $\begin{aligned} \mathrm{g}=\mathbf{0 . 1 7 5} ; \mathrm{SE} & =0.034 ; \mathbf{p}<.0001 \\
\tau^{2} & =.001\end{aligned}$ & $\begin{aligned} \mathrm{g}=\mathbf{0 . 1 7 0} ; \mathrm{SE} & =0.032 ; \mathbf{p}<\mathbf{. 0 0 0 1} \\
\boldsymbol{\tau}^{2} & =\mathbf{0 . 0 0 0 2}\end{aligned}$ \\
\hline $\begin{array}{l}\text { Moderator } \\
\text { Randomization }\end{array}$ & $p=.194^{1}$ & $p=.185^{2}$ \\
\hline
\end{tabular}

Note: $m$ refers to the number of studies, $k$ refers to the number of effect sizes. Note that for the influential case analysis on all studies (Table 2 right), the moderators randomization and type of control did not reach significance $(p=.48$ and $p=.06$, respectively), but we reported the non-active control group studies data separately for comparison with Table 1 and Table 2 left. See Supplementary Material for Bayesian analyses. We used S\&G2020's R script and data file, available at https://osf.io/rquye. Supplementary Table 1 lists the studies excluded at the various analysis steps.

${ }^{1}$ All three moderators (i.e., randomization, baseline, age) were entered in one moderator analysis. When three separate analyses were ran, then the moderator randomization was at $p=.158$.

${ }^{2}$ All three moderators (i.e., randomization, baseline, age) were entered in one moderator analysis. When three separate analyses were ran, then the moderator randomization was at $p=.157$. 
Table 3: Reanalysis of the data set of Sala \& Gobet (2020) with a new approach (see Sections 4.2 and 4.3 for details)

\begin{tabular}{|c|c|c|c|}
\hline & $\begin{array}{l}\text { Removal of near-transfer active } \\
\text { control group studies }\end{array}$ & $\begin{array}{l}\text { Additional removal of studies } \\
\text { without pre-tests }\end{array}$ & $\begin{array}{l}\text { Additional removal of studies without pre-tests, } \\
\text { combined with influential case analyses ( } 7 \text { effect } \\
\text { sizes) }\end{array}$ \\
\hline & $m=51 ; k=233$ & $m=42 ; k=197$ & $m=41 ; k=190$ \\
\hline RVE & $\begin{aligned} \mathbf{g}=\mathbf{0 . 2 0 8} ; \mathrm{SE}=0.043 ; \mathbf{p}<. \mathbf{0 0 0 1} \\
\mathrm{df}=36.99 ; 95 \% \text { CI }[0.121 ; 0.295] \\
\boldsymbol{\tau}^{\mathbf{2}}=\mathbf{0 . 0 4 4} ; \mathbf{I}^{\mathbf{2}}=\mathbf{4 5 . 8 1 \%}\end{aligned}$ & $\begin{aligned} \mathbf{g}=\mathbf{0 . 2 4 3} ; \mathrm{SE}=0.052 ; \mathbf{p}<\mathbf{. 0 0 0 1} \\
\mathrm{df}=34.41 ; 95 \% \text { CI }[0.136 ; 0.345] \\
\boldsymbol{\tau}^{\mathbf{2}}=\mathbf{0 . 0 5 9} ; \mathbf{I}^{\mathbf{2}}=\mathbf{3 6 . 4 2 \%}\end{aligned}$ & $\begin{aligned} & \mathbf{g}=\mathbf{0 . 2 3 4} ; \mathrm{SE}=0.046 ; \mathbf{p}<.0001 \\
& \mathrm{df}= 28.84 ; 95 \% \text { CI }[0.141 ; 0.327] \\
& \boldsymbol{\tau}^{\mathbf{2}}=\mathbf{0 . 0 1 7} ; \mathbf{I}^{\mathbf{2}}=\mathbf{1 3 . 0 3 \%}\end{aligned}$ \\
\hline $\mathrm{RE}$ & $\begin{array}{c}\mathbf{g}=\mathbf{0 . 1 9 5} ; \mathrm{SE}=0.039 ; \mathbf{p}<.0001 \\
\boldsymbol{\tau}^{\mathbf{2}}=\mathbf{0 . 0 3 3}\end{array}$ & $\begin{array}{c}\mathbf{g}=\mathbf{0 . 2 2 6} ; \mathrm{SE}=0.045 ; \mathbf{p}<\mathbf{. 0 0 0 1} \\
\boldsymbol{\tau}^{2}=\mathbf{0 . 0 3 7}\end{array}$ & $\begin{array}{c}\mathrm{g}=\mathbf{0 . 2 1 3} ; \mathrm{SE}=0.041 ; \mathbf{p}<.0001 \\
\tau^{2}=\mathbf{0}\end{array}$ \\
\hline
\end{tabular}

Note: $\mathrm{m}$ refers to the number of studies, $\mathrm{k}$ refers to the number of effect sizes. Note that for these three analyses, the moderators randomization and type of control did not reach significance (see main text for details). For comparison purpose with S\&G's analyses, we present separate analyses for the non-active and active control group studies respectively in the Supplementary Material. We used S\&G2020's R script and data file, available at https://osf.io/rquye. Supplementary Table 1 lists the studies excluded at the various analysis steps. 


\section{Supplementary material}

\section{Bayesian analyses}

In addition to RVE et RE analyses, $\mathrm{S} \& \mathrm{G} 2020$ reported Bayesian analyses. For completion, we used their program (available at https://osf.io/rquye) to also run Bayesian analyses on our analyses reported in the main text. S\&G 2020 "estimated the Bayes factors (BFs) for two sets of competing hypotheses for $\mathrm{g}$ and $\tau$. First, [they] compared the alternative hypothesis H1: $\mathrm{g}=10$ with the null hypothesis H0: $\mathrm{g}=0$. Second, [they] compared the alternative hypothesis H1: $\tau>0$ with the null hypothesis H0: $\tau=0$. BFs $>1$ indicated support for H1, while BFs $<1$ indicated support for H0. In line with common guidelines, H1 was considered as substantially supported only if BF $>3$ (i.e., H1 three times more likely to be true than H0; e.g., Dougherty, Hamovitz, \& Tidwell, 2016). Analogously, H0 was substantially supported only if BF < 0.333 (i.e., H0 three times more likely to be true than H1)." (Sala \& Gobet, 2020, page 1434). We used S\&G2020's distribution assumptions and priors.

S\&G2020 based their priors on Sala et al.'s (2019) second-order meta-analysis, notably expecting "the mean effect size to be null (prior $g=0$ ) in models including active control groups and slightly positive (prior $\mathrm{g}=0.150$ ) in models including passive controls groups" (Sala \& Gobet, 2020, page 1434).

Table A. Bayesian analyses for the analyses presented in Table 2 and the main text

\begin{tabular}{llll}
\hline & & $B F g$ & $B F \tau$ \\
\hline Table 2 left & Step 2 (all 9 influential cases removed); Non-active control group studies & 261,108 & 0.007 \\
Table 2 right & Influential case analysis on all studies (main analysis data); Non active & & \\
& control group studies & 254,736 & 0.007 \\
\hline Main text & Near-transfer phonological vs. musical training & 0.447 & 0.010 \\
& Near-transfer phonological vs. musical training without Herrera et al. & 0.383 & 0.012 \\
\hline
\end{tabular}

Dougherty, M. R., Hamovitz, T., \& Tidwell, J. W. (2016). Reevaluating the effectiveness of n-back training on transfer through the Bayesian lens: Support for the null. Psychonomic Bulletin \& Review, 23, 206- 316. https://doi.org/10.3758/s 13423-015-0865-9

Sala, G., Aksayli, N. D., Tatlidil, K. S., Tatsumi, T., Gondo, Y., \& Gobet, F. (2019). Near and far transfer in cognitive training: A second- order meta-analysis. Collabra: Psychology, 5, 18. https://doi.org/10. 1525/collabra.203 


\section{Complementary analyses to Table 3}

For the three analyses reported in Table 3 ("Reanalysis of the data set of Sala \& Gobet (2020) with a new approach"), the moderators randomization and type of control did not reach significance (see main text for details). For comparison purpose with S\&G2020, we also analyzed the non-active and active control group studies separately. We used S\&G2020's R script and data file, available at https://osf.io/rquye. Our Supplementary Table 1 reproduces the data file of S\&G2020 with additional information about the studies excluded at the various analysis steps. It is openly available in OSF at https://osf.io/w5kx9/. Below, we also present Bayesian analyses for these three analyses (see Section 1 above for details of the implementation of the Bayesian analyses of S\&G2020).

\section{Table B. Complementary analyses to Table 3 of the main text}

\begin{tabular}{|c|c|c|c|}
\hline & $\begin{array}{l}\text { Removal of near-transfer active } \\
\text { control group studies }\end{array}$ & $\begin{array}{l}\text { Additional removal of studies } \\
\text { without pre-tests }\end{array}$ & $\begin{array}{l}\text { Additional removal of studies without pre-tests, } \\
\text { combined with influential case analyses ( } 7 \text { effect } \\
\text { sizes) }\end{array}$ \\
\hline & $\begin{array}{l}\text { Non active control group studies (= } \\
\text { Sala \& Gobet's main analysis, see } \\
\text { Table 1) }\end{array}$ & $\begin{array}{l}\text { Non-active control group } \\
\text { studies: }\end{array}$ & Non-active control group studies: \\
\hline & $m=41 ; k=144$ & $m=33 ; k=114$ & $m=32 ; k=110$ \\
\hline RVE & $\begin{array}{l}\mathbf{g}=\mathbf{0 . 2 2 8} ; \mathrm{SE}=0.045 ; \mathbf{p}<\mathbf{. 0 0 1} \\
\mathrm{df}=30.1 ; 95 \% \text { CI }[0.137 ; 0.320] \\
\boldsymbol{\tau}^{\mathbf{2}}=\mathbf{0 . 0 4 2} ; \mathbf{I}^{\mathbf{2}}=\mathbf{4 3 . 1 1 \%}\end{array}$ & $\begin{array}{l}\mathbf{g}=\mathbf{0 . 2 5 1} ; \mathrm{SE}=0.054 ; \mathbf{p}<\mathbf{. 0 0 0 1} \\
\mathrm{df}=25.30 ; 955 \text { CI }[0.140 ; 0.362] \\
\boldsymbol{\tau}^{2}=\mathbf{0 . 0 4 0} ; \mathbf{I}^{2}=\mathbf{2 8 . 0 7 \%}\end{array}$ & $\begin{array}{l}\mathbf{g}=\mathbf{0 . 2 4 2} ; \text { SE }=0.056 ; \mathbf{p}<\mathbf{. 0 0 0 1} \\
\mathrm{df}=25.00 ; 95 \% \text { CI }[0.126 ; 0.357] \\
\boldsymbol{\tau}^{2}=\mathbf{0 . 0 3 2} ; \mathbf{I}^{\mathbf{2}}=\mathbf{2 1 . 9 1 \%}\end{array}$ \\
\hline $\mathrm{RE}$ & $\begin{array}{l}\mathbf{g}=\mathbf{0 . 2 0 1} ; \mathrm{SE}=0.041 ; \mathbf{p}<.001 \\
\boldsymbol{\tau}^{2}=\mathbf{0 . 0 2 3}\end{array}$ & $\begin{array}{l}\mathbf{g}=\mathbf{0 . 2 2 9} ; \mathrm{SE}=0.045 ; \mathbf{p}<.0001 \\
\boldsymbol{\tau}^{2}=\mathbf{0 . 0 0 8}\end{array}$ & $\begin{array}{l}\mathbf{g}=\mathbf{0 . 2 1 9} ; \mathrm{SE}=0.049 ; \mathbf{p}<.0001 \\
\tau^{2}=\mathbf{0 . 0 0 5}\end{array}$ \\
\hline $\begin{array}{l}\text { Moderator } \\
\text { Randomization }\end{array}$ & $p=.487$ & $p=.453 *$ & $p=.337 *$ \\
\hline & Active control group studies & Active control group studies & Active control group studies \\
\hline & $\mathrm{m}=18 ; \mathrm{k}=89$ & $\mathrm{~m}=17 ; \mathrm{k}=83$ & $\mathrm{~m}=17 ; \mathrm{k}=80$ \\
\hline RVE & $\begin{array}{l}\mathbf{g}=\mathbf{0 . 1 2 5} ; \mathrm{SE}=.075 ; \mathbf{p}=\mathbf{. 1 2 1} \\
\mathrm{df}=12.35 ; 95 \% \text { CI }[-0.038 ; 0.287] \\
\boldsymbol{\tau}^{2}=\mathbf{0 . 0 4 6} ; \mathbf{I}^{\mathbf{2}}=\mathbf{3 5 . 5 9 \%}\end{array}$ & $\begin{array}{l}\mathbf{g}=\mathbf{0 . 1 5 2} ; \mathrm{SE}=0.084 ; \mathbf{p}=\mathbf{. 0 9 4} \\
\mathrm{df}=14.06 ; 95 \% \text { CI }[-0.294 ; 0.333] \\
\boldsymbol{\tau}^{2}=\mathbf{. 0 6 5} ; \mathbf{I}^{\mathbf{2}}=\mathbf{3 4 . 2 3 \%}\end{array}$ & $\begin{array}{l}\mathbf{g}=\mathbf{0 . 1 6 8} ; \text { SE }=0.051 ; \mathbf{p}=\mathbf{. 0 0 9} \\
\mathrm{df}=9.71 ; 95 \% \text { CI }[0.053 ; 0.282] \\
\boldsymbol{\tau}^{\mathbf{2}}=\mathbf{0} ; \mathbf{I}^{\mathbf{2}}=\mathbf{0 \%}\end{array}$ \\
\hline RE & $\begin{array}{l}\mathbf{g}=\mathbf{0 . 1 4 7} ; \mathrm{SE}=0.072 ; \mathbf{p}=\mathbf{. 0 4 1} \\
\boldsymbol{\tau}^{2}=\mathbf{0 . 0 4 4}\end{array}$ & $\begin{array}{l}\mathbf{g}=\mathbf{0 . 1 8 4} ; \mathrm{SE}=0.095 ; \mathbf{p}=\mathbf{. 0 5 2} \\
\boldsymbol{\tau}^{2}=\mathbf{0 . 0 6 4}\end{array}$ & $\begin{array}{l}\mathbf{g}=\mathbf{0 . 1 7 2} ; \mathrm{SE}=0.071 ; \mathbf{p}=\mathbf{. 0 1 5} \\
\boldsymbol{\tau}^{2}=\mathbf{0}\end{array}$ \\
\hline
\end{tabular}


Note: $m$ refers to the number of studies, $\mathrm{k}$ refers to the number of effect sizes. The grey-shaded area corresponds to Sala \& Gobet's findings (see Table 1 main text).

* All three moderators (i.e., randomization, baseline, age) were entered in one moderator analysis.

Table C. Bayesian analyses to the analyses presented in Table B (see Section 1 above for details of the implementation of the Bayesian analyses of S\&G2020)

\begin{tabular}{lll}
\hline & $\mathrm{BFg}$ & $\mathrm{BF} \tau$ \\
\hline $\begin{array}{l}\text { Removal of near-transfer active control group studies } \\
\quad \text { - Active control group studies }\end{array}$ & 12.871 & 0.200 \\
$\begin{array}{l}\text { Additional removal of studies without pre-tests } \\
\text { - Non active control group studies }\end{array}$ & $1.226609 \mathrm{e}+07$ & 0.014 \\
- Active control group studies & 70.801 & 0.154 \\
\hline Combined with influential case analyses & & \\
- Non active control group studies & 864,078 & 0.011 \\
- Active control group studies & 145.591 & 0.00553 \\
\hline
\end{tabular}


